Received: 2019.06.29 Accepted: 2019.08.21 Published: 2019.10.28

\title{
A Rare Case of Small Bowel Obstruction Due to Paraduodenal Hernia
}

Authors' Contribution:

Study Design A

Data Collection B

Statistical Analysis C

Data Interpretation D

Manuscript Preparation E

Literature Search F

Funds Collection G

Corresponding Author:

Conflict of interest:
EF 1 Rahaf Suhail Al Otaibi

E 1 Huda S. Al Maghrabi

F 2 Yazeed Bin Dous

E 1 Haifaa Malaekah
1 Surgical Department, King Abdullah Bin Abdulaziz University Hospital, Princess Nourah Bint Abdulrahman University, Riyadh, Saudi Arabia

2 Department of Medical Imaging, King Abdullah Bin Abdulaziz University Hospital, Princess Nourah Bint Abdulrahman University, Riyadh, Saudi Arabia

Haifaa Malaekah, e-mail: h_malaika@hotmail.com

None declared

\begin{abstract}
Patient: Female, 24
Final Diagnosis: Paraduodenal hernia

Symptoms: Abdominal pain • Bowel obstruction

Medication: -

Clinical Procedure: Surgical excision of the sac and release of adhesion

Specialty: Surgery

Objective: Challenging differential diagnosis

Background: Paraduodenal hernia, or mesocolic hernia, is a rare congenital form of internal abdominal hernia, and is classified into right and left, with left paraduodenal hernia being more common than right. Patients can have nonspecific symptoms and signs, which make the diagnosis challenging. However, patients may present with obstruction or ischemia requiring emergency surgery. This report is of a case of left paraduodenal hernia in a 24-year-old woman who presented with acute abdominal pain at two-weeks post-partum and who was managed with open surgery with good clinical outcome.

Case Report: A 24-year-old Saudi woman presented with recurrent chronic abdominal pain that was managed conservatively for the previous six years. At two-weeks post-partum, she presented with acute abdominal pain, vomiting, and constipation. Computed tomography (CT) of the abdomen showed a left paraduodenal hernia. The patient underwent timely open laparotomy due to the presence of bowel dilatation. Her postoperative recovery was uneventful.

Conclusions: Although rare, this case has shown that paraduodenal hernia can cause intestinal obstruction. CT imaging increases the chance of early diagnosis and timely surgery. Although laparoscopic surgical repair may be preferable in some cases, this patient underwent successful open repair.
\end{abstract}

MeSH Keywords: Congenital Abnormalities • Hernia, Abdominal • Intestinal Obstruction

Full-text PDF: https://www.amjcaserep.com/abstract/index/idArt/918403

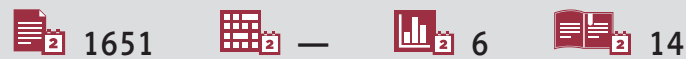




\section{Background}

An internal abdominal hernia is a projection of abdominal viscera through a defect in the peritoneum or mesentery $[1,2]$. The defect can be acquired or congenital, with acquired peritoneal or mesenteric defects caused by surgery, including bariatric surgery such as Roux-en-Y gastric bypass [1,2]. Congenital internal abdominal hernia results from abnormal embryonic rotation of the midgut that persists after birth [1]. In 1984, Ghahremani [1] classified congenital internal abdominal herniation into paraduodenal hernia (50-55\%), pericecal hernia (10-15\%), transmesenteric hernia (8-10\%), hernia of the foramen of Winslow (6-10\%), intersigmoid hernia (4-8\%), and paravesical hernia (<4\%) [1]. Radiological studies, including abdominal computed tomography (CT) imaging, have shown that paraduodenal hernia can be classified into the right (25\%) and left $(75 \%)[1,2]$. Left-sided paraduodenal hernias consist of small bowel loops in the left paraduodenal fossa of Landzert, and right-sided paraduodenal hernias consist of small bowel loops in the paraduodenal fossa of Waldayer [2,3].

In a recent literature review of previously published cases, Schizas et al. showed that although paraduodenal hernia accounted for $53 \%$ of all internal hernias, they caused between $0.2-0.9 \%$ of all cases of intestinal obstruction [3]. Paraduodenal hernia is either asymptomatic, or presents with nonspecific symptoms such as chronic abdominal pain, digestive disorders, but can result in bowel obstruction that, if undiagnosed and treated surgically, can result in bowel perforation [4].

This report is of a case of left paraduodenal hernia in a 24-yearold woman who presented with acute abdominal pain at twoweeks post-partum who was diagnosed with abdominal CT and managed with open surgery with good clinical outcome.

\section{Case Report}

A 24-year-old Saudi woman presented to the emergency department with a three-hour history of acute colicky abdominal pain in the epigastric and left upper abdominal quadrant. The pain had started six days before her arrival at the emergency department and increased gradually. On admission, she had abdominal distension and non-projectile bilious vomiting. She complained of constipation and flatus during the 24 hours before hospital admission. Two weeks previously, she had undergone a normal spontaneous vaginal delivery.

In her past medical history, she had similar episodes of abdominal pain since childhood, and two previous episodes required hospitalization. During each of her previous two hospital admissions, she had been managed conservatively, without a definite diagnosis. She had no other previous medical or surgical history.
On physical examination, her vital signs were within normal limits. Her abdomen was distended and tender on palpation on the left side with mild distension. Routine laboratory tests were within normal limits.

Contrast-enhanced abdominal computed tomography (CT) showed the typical features of left paraduodenal hernia (Figures 1, 2). The CT findings showed a few small bowel loops that herniated into the left paraduodenal fossa of Landzert in the inferior and lateral aspect of the ascending part of the duodenum and the posterior and lateral aspect of the inferior mesenteric vein. The herniated bowel loops formed an encapsulated mass with engorged mesenteric vessels around the small bowel and hernia orifice (Figures 1, 2).

In 2015, the patient experienced similar symptoms and was admitted to hospital, but was managed conservatively and improved. A retrospective review of her previous abdominal CT scan images showed the presence of a left paraduodenal hernia that had not been previously diagnosed (Figure 3).

After giving informed consent, the patient was taken to the operating theater for exploratory laparotomy. The abdomen was opened through a supraumbilical midline incision. A mild increase in fluid was found in the abdomen. The appendix, cecum, omentum, and transverse colon were retracted to the left side. A large peritoneal sac was found (Figure 4), with most of the small bowel inside the sac, with distal ileum outside. The bowel initially appeared dusky and congested. Further assessment of the pouch-like structure showed a neck measuring approximately $2 \mathrm{~cm}$ in diameter, located to the left of the fourth part of the duodenum and duodenojejunal junction (Figure 5). There was a difficulty in removing the bowel from the peritoneal sac. Therefore, a decision was made to open the sac and the neck of the sac in an avascular plane. After a few minutes, the small bowl regained its normal color, and the hernia sac was excised completely. Final examination of the small bowel and large bowel before surgical closure was unremarkable, and examination of the peritoneal cavity showed no other abnormalities. The small bowel was reduced back in the peritoneal cavity, and irrigation with warm saline was performed before the abdomen was closed in layers. A transversus abdominis plane block for postoperative pain control was performed under ultrasound guidance.

The patient's postoperative course was unremarkable, and she was able to open her bowels on the first postoperative day, tolerated oral feeding, and she tolerated the pain. Postoperative laboratory tests were unremarkable. She was discharged home on the fourth postoperative day in good general condition and was seen four weeks later in the outpatient department where review showed a healing surgical incision and no complications. 


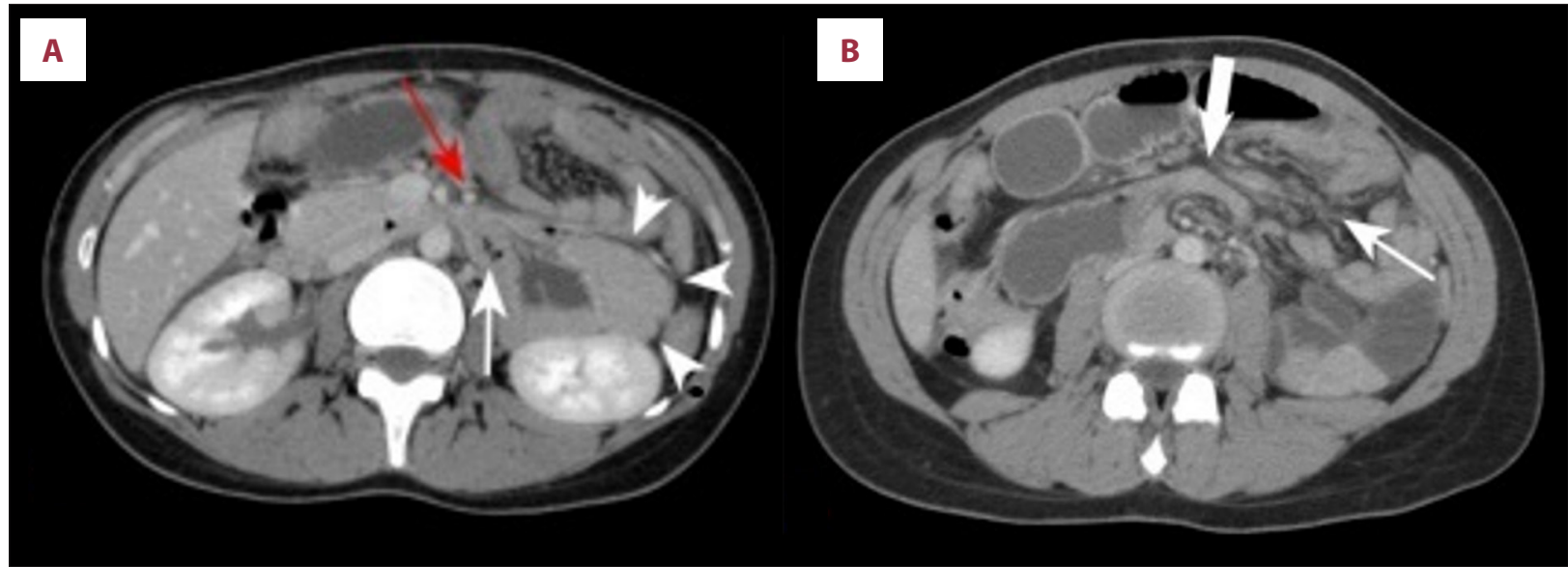

Figure 1. Axial contrast-enhanced abdominal computed tomography (CT) images of the left paraduodenal hernia in a 24-yearold woman. (A) Contrast-enhanced abdominal CT scan shows a mass of small bowel loops of jejunum, some of which are dilated. The arrow heads show the inferior mesenteric vein anterior and medial to herniated bowel loops (red arrow) and the ascending part of the duodenum (white arrow). (B) Contrast-enhanced abdominal CT scan shows the third part of the duodenum (thick white arrow), the mesenteric pedicle of the herniated bowel (white arrow).

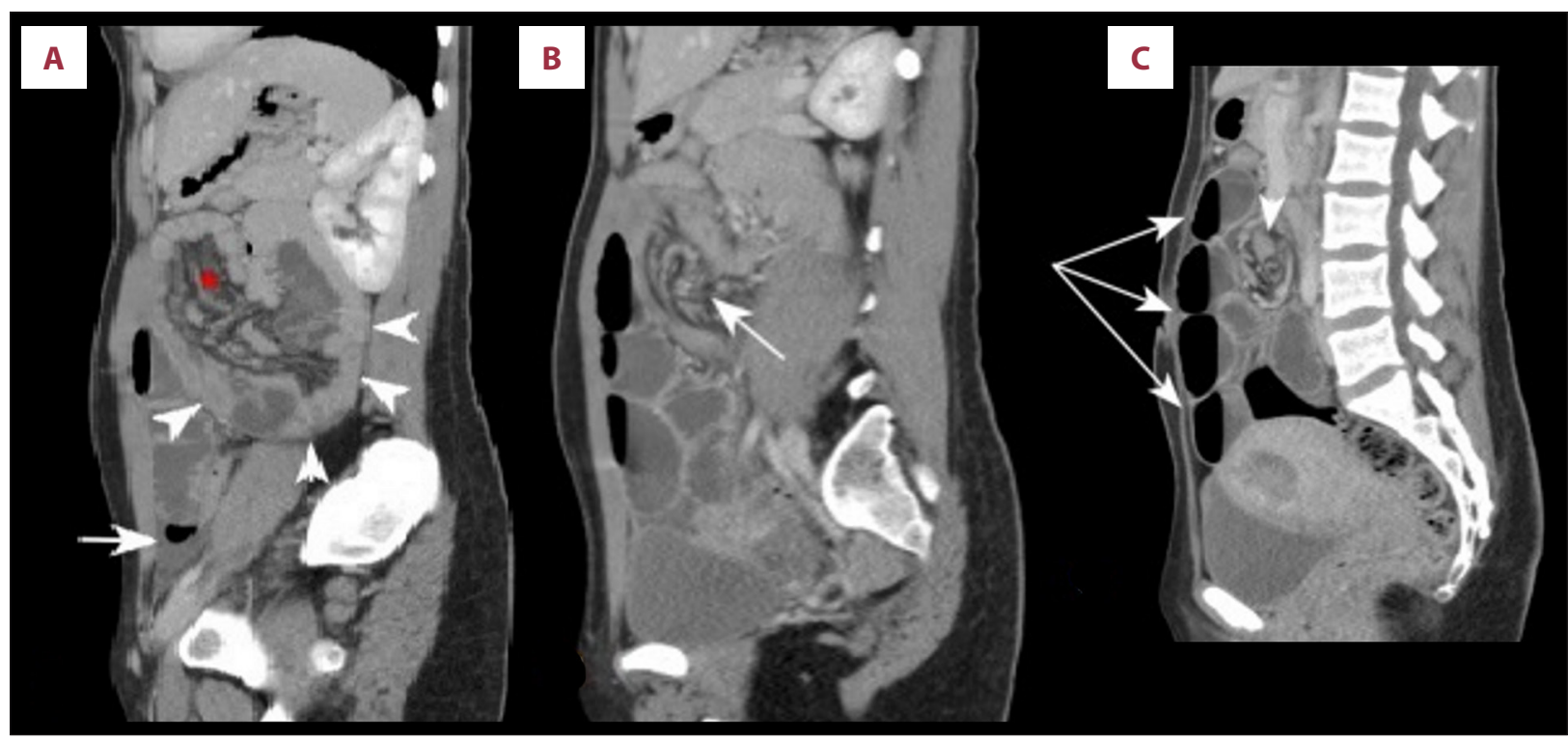

Figure 2. Sagittal contrast-enhanced abdominal computed tomography (CT) images of the left paraduodenal hernia in a 24-year-old woman. (A) Contrast-enhanced abdominal CT scan shows a mass of small bowel loops (arrowheads), with engorgement of the mesenteric vessels (red asterisk), and mild pelvic ascites (white arrow). (B) Contrast-enhanced abdominal CT scan shows a mass of mesenteric vessels (white arrow). (C) Contrast-enhanced abdominal CT scan shows the transition zone of the small bowel (arrowhead), and multiple dilated small bowel loops with air-fluid levels (white arrows).

\section{Discussion}

Paraduodenal hernia is rare, with fewer than 200 cases reported in the literature [3]. Paraduodenal hernia is the most common type of internal abdominal hernia but is a cause of intestinal obstruction in less than $0.9 \%$ of cases [3]. The congenital defect associated with paraduodenal hernia occurs during embryonic development of the midgut when the pre-arterial portion fails to rotate normally and becomes trapped behind the colonic mesentery to create a peritoneal pocket containing the small bowel and the distal ileum [4]. Left paraduodenal hernia occurs in the abnormal fossa of Landzert, which is a defect in the left transverse mesocolon and descending mesocolon and represents the majority of cases of paraduodenal hernia [2-5]. However, right paraduodenal hernia is located behind the ascending mesocolon and right side of transverse mesocolon in Waldeyer's fossa (Figure 6) $[5,6]$. 


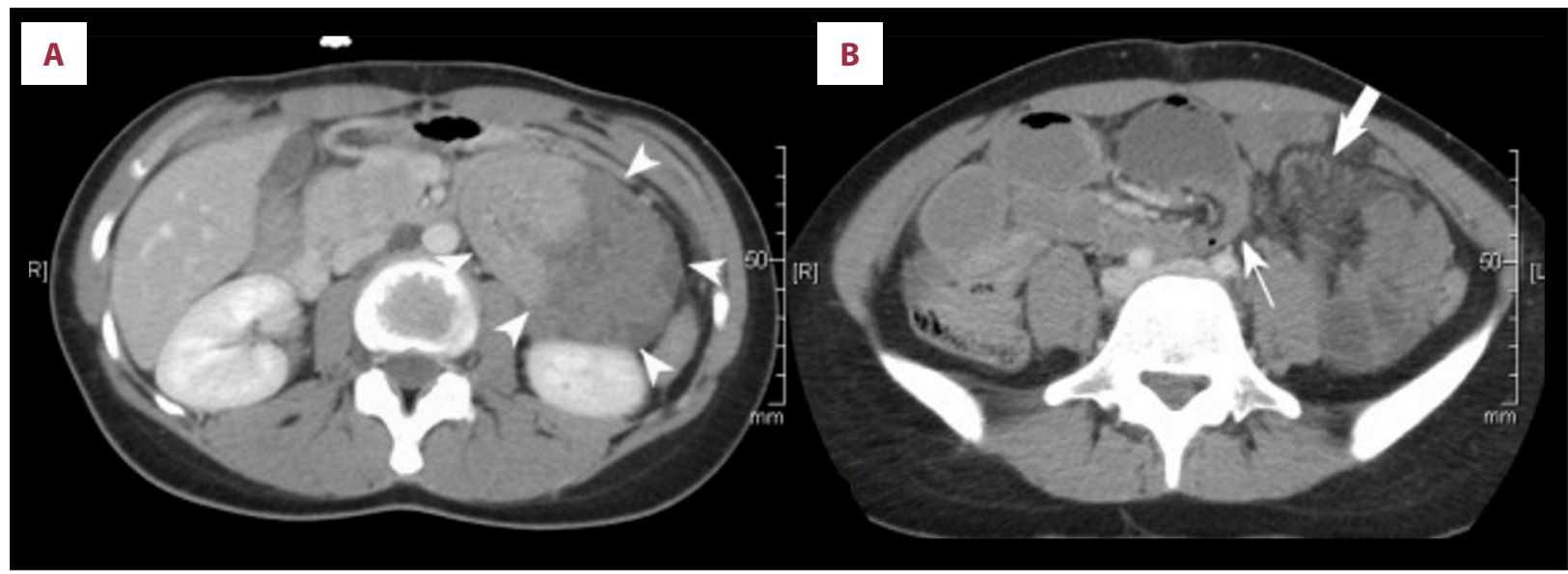

Figure 3. Review of the axial contrast-enhanced abdominal computed tomography (CT) images from 2015, from the same patient. (A) Contrast-enhanced abdominal CT scan (2015) shows a mass containing small bowel loops (arrowheads). (B) Contrastenhanced abdominal CT scan (2015) shows engorged mesenteric vessels (thick white arrow) and the transition zone of the small bowel (white arrow).

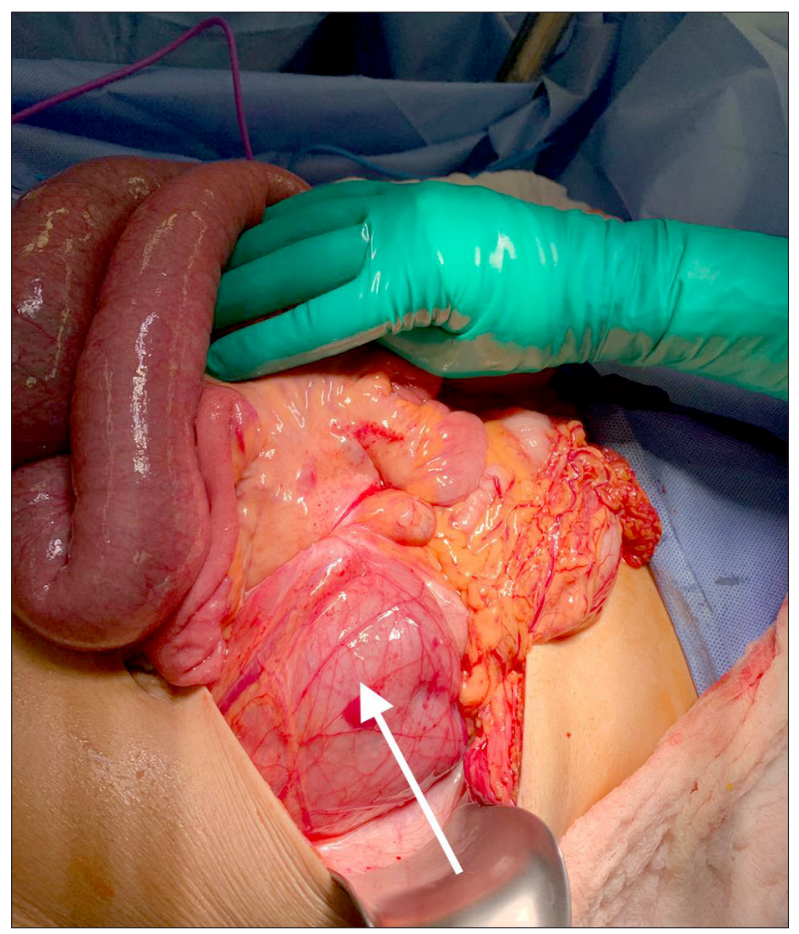

Figure 4. Intra-operative findings during open surgery for a left paraduodenal hernia in a 24-year-old woman. Small bowel loops are present inside the hernia sac (white arrow).

The clinical presentation of paraduodenal hernia is variable. In the review of 159 published cases of paraduodenal hernia conducted by Schizas et al. in 2019 , the most common presentation was abdominal pain (96.7\%), followed by vomiting (66.7\%), nausea (34\%), signs of bowel obstruction (32.7\%), and fever $(6.5 \%)$, with no differences in presentation between the left and right paraduodenal hernia [3]. The mean age at

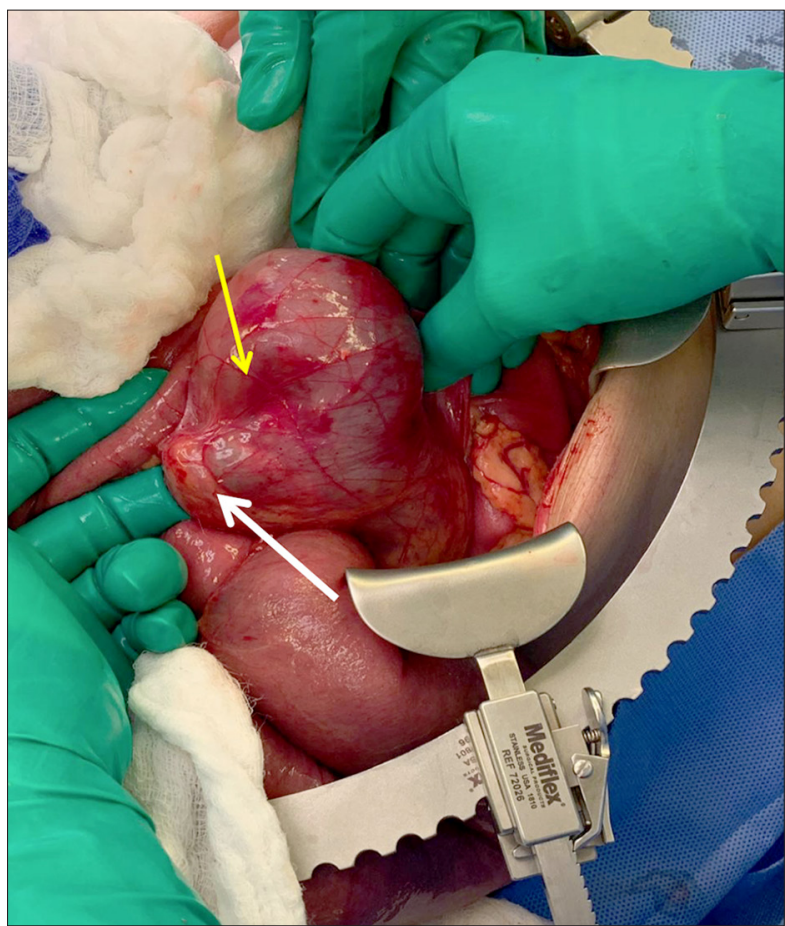

Figure 5. Intra-operative findings during open surgery for a left paraduodenal hernia in a 24-year-old woman. The left paraduodenal hernia sac is shown (white arrow) and the neck of the hernia sac is shown. The peritoneal membrane is shown that forms the hernia pouch (yellow arrow).

diagnosis was found to be 44.1 years with a female to male ratio of 1: 2 [3]. Other studies have reported the most common age at diagnosis of between the fourth and sixth decade $[3,7]$. The patient in this report was 24-years-old and female. 


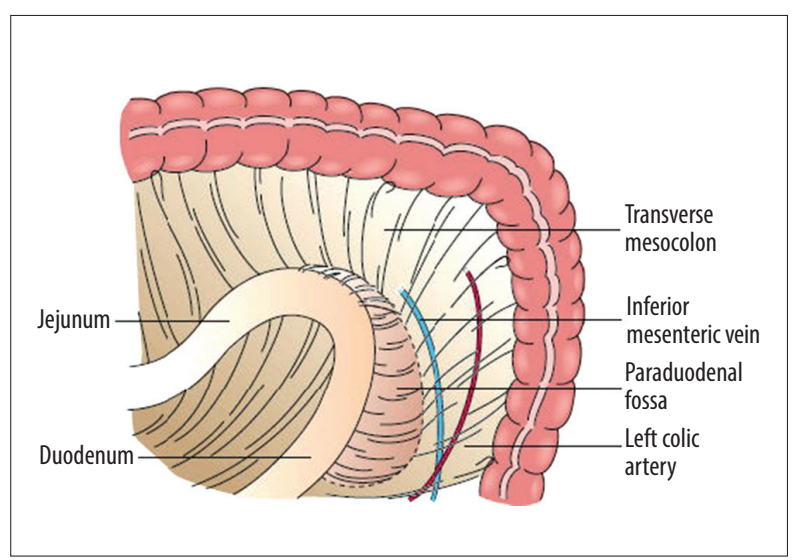

Figure 6. Schematic diagram showing the anatomic relationships of the paraduodenal fossa and the transverse mesocolon. Figure obtained with permission from: Mulholland MW, Ed. Greenfield's Surgery: Scientific Principles and Practice. Revised $6^{\text {th }}$ Edition. 2016. Wolters Kluwer Health. (ISBN: 9781469890012).

The preoperative diagnosis of internal abdominal hernia, including paraduodenal hernia, can be challenging due to the nonspecific clinical presentation [8,9]. However, accurate preoperative diagnosis of paraduodenal hernia is important because of the potentially serious complications. Contrast-enhanced abdominal computed tomography (CT) imaging has an important role in preoperative diagnosis of internal hernias with a reported sensitivity of $94-100 \%$ and an accuracy of $90-95 \%$ [5,10]. CT images in case of left paraduodenal hernia show a cluster of bowel loops at the same level of the duodenojejunal junction, between the stomach and the pancreas, or behind the descending colon [7]. In cases of left paraduodenal hernia, angiography of the superior mesenteric artery can identify displaced jejunal arteries superiorly and to the left [11]. The CT findings for right paraduodenal hernia identify encapsulated small bowel behind the hepatic flexure of the colon [5]. Barium meal can give also identify and provide a definitive diagnosis of paraduodenal hernia preoperatively $[6,11]$. In cases of left paraduodenal hernia on barium meal, the small bowel fills with barium will be in an area on the left side of the midline above the ligament of Treitz, while the barium in a right paraduodenal hernia will be on the right of the midline $[6,12]$. Barium-enhanced studies, abdominal ultrasonography, and angiography are imaging modalities that confirm the diagnosis of paraduodenal hernia, with a barium upper gastrointestinal series, and abdominal CT providing a more accurate diagnosis [13]. In this case, the patient was diagnosed preoperatively by abdominal
$\mathrm{CT}$, which showed the typical small bowel features. However, the $\mathrm{CT}$ findings were similar to the previous scan that had been previously performed in 2015 at another healthcare facility, which highlights the importance of recognizing and correctly diagnosing symptomatic paraduodenal hernia.

Paraduodenal hernia is associated with a high patient morbidity of $14.5 \%$, and a preoperative mortality rate of $1.4 \%$ [3]. The optimal treatment is surgical correction because of the high risk of developing hernial incarceration in $50 \%$ of untreated cases [7]. Surgical correction of paraduodenal hernia consists of a reduction of the small bowel and closure of the hernial opening [4]. An alternative approach is widening the hernial opening and dividing the inferior mesenteric vessels $[7,14]$. The laparoscopic approach has advantages over the open approach, including less postoperative pain, more rapid postoperative mobilization, a small surgical scar, shorter hospital stay, and reduced postoperative morbidity $[3,8]$. This patient was managed by an open surgical approach due to the presence of bowel dilatation. The patient had good postoperative recovery with no complications and was discharged from hospital after four days with no complications at routine outpatient follow-up.

\section{Conclusions}

A case is reported of left paraduodenal hernia in a 24-yearold woman who presented with acute abdominal pain at twoweeks post-partum and who was managed with open surgery with good clinical outcome. Although rare, this case has shown that paraduodenal hernia can cause intestinal obstruction. Late presentation or delay in diagnosis may lead to bowel perforation and peritonitis, which increases the risk of patient mortality. As this case has shown, patients may present with a history of chronic abdominal pain. Computed tomography (CT) of the abdomen shows the diagnostic features of bowel within the lesser sac at the level of the duodenojejunal junction, between the pancreas and stomach, or behind the descending colon. Therefore, $\mathrm{CT}$ imaging increases the chance of early diagnosis and timely surgery. Although laparoscopic surgical repair may be preferable in some cases, this patient underwent successful open repair.

\section{Conflict of interest}

None. 


\section{References:}

1. Ghahremani GG: Internal abdominal hernias. Surg Clin North Am, 1984; 64: 393-406

2. Mathieu D, Luciani A: Internal abdominal herniations. Am J Roentgenol, 2004; 183(2): 397-404

3. Schizas D, Apostolou K, Krivan S et al: Paraduodenal hernias: A systematic review of the literature. Hernia, 2019 [Epub ahead of print]

4. Bartlett MK, Wang C, Williams WH: The surgical management of paraduodenal hernia. Ann Surg, 1968; 168(2): 249-54

5. Dayananda L, Sreekumar KP, Moorthy S, Prabhu NK: Para duodenal hernias - a pictorial essay. Indian J Radiol Imaging, 2006; 16(4): 469-71

6. Khan MA, Lo AY, Vande Maele DM: Paraduodenal hernia. Am Surg, 1998; 64(12): 1218-22

7. Muneer $\mathrm{H}$, Jawad A, Al Aradi J: Left paraduodenal hernia. Bahrain Med Bull, 2017; 39(4): 250-52
8. Doishita S, Takeshita T, Uchima Y, Uchima Y et al: Internal hernias in the era of multidetector CT: Correlation of imaging and surgical findings. Radiographics, 2016; 36(1): 88-106

9. Takeyama N, Gokan T, Ohgiya Y et al: CT of internal hernias. Radiographics, 2005; 25(4): 997-1015

10. Selcuk D, Kantarci F, Oğüt G, Korman U: Radiological evaluation of internal abdominal hernias. Turk J Gastroenterol, 2005; 16(2): 57-64

11. Kuzinkovas V, Haghighi K, Singhal R, Andrews N: Paraduodenal hernia: A rare cause of abdominal pain. Can J Surg, 2008; 51(6): E127-28

12. El Sharkawy MS, Al-Nakshabandi N: Barium meal followthrough, and CT findings in paraduodenal hernia. Ann Saudi Med, 2007; 27(6): 450-52

13. Hassani K, Aggouri Y, Laalim S et al: Left paraduodenal hernia: A rare cause of acute abdomen. Pan Afr Med J, 2014; 17: 230

14. Bouchentouf SM, Raissouni F, El Kaoui $\mathrm{H}$ et al: Intestinal obstruction due to a left paraduodenal hernia: A case report. J Med Case Rep, 2013; 7: 272 